\title{
Resultados a largo plazo de la microdiscectomía lumbar en una población labo- ralmente activa
}

\author{
J.V. Martínez Quiñones; J. Aso; F. Consolini y R. Arregui
}

Servicio de Neurocirugía. Hospital MAZ. Zaragoza.

\section{Resumen}

Introducción. La microdiscectomía constituye un procedimiento estándar en el tratamiento de la hernia discal lumbar (HDL). En este estudio se ha analizado el resultado clínico de la microdiscectomía lumbar en una muestra de pacientes laboralmente activa, intervenidos en nuestro servicio.

Material y métodos. Análisis retrospectivo y seguimiento durante 5 años, de una muestra de 142 pacientes intervenidos mediante microdiscectomía lumbar durante el bienio 2004-2005. El resultado clínico se evaluó en base al esquema propuesto por Herron y Turner, que evalúa la reducción del dolor, el uso de fármacos, la restricción de las actividades físicas y el regreso a la vida laboral.

Resultados. 116 hombres y 26 mujeres, con edades medias de 37,9 y 45,4 años, respectivamente, fueron intervenidos por HDL. Clínicamente la ciatalgia predominó sobre la lumbalgia en una proporción de 3 a 1. El nivel discal L5-S1 fue intervenido en el $68,3 \%$ de los casos. Se consideró el origen del padecimiento como accidente de trabajo en 107 pacientes $(75,3 \%)$. En el $44,3 \%$ de la serie además del disco sintomático, existía un disco adicional afecto. Se apreció una evolución postquirúrgica inicial desfavorable en $\mathbf{4 2}$ pacientes (33\%), 15 de los cuales se recuperaron en el plazo de 3 meses, y otros quince a lo largo de un año. Fue preciso reintervenir a 16 pacientes por recidiva discal $(11 \%)$. La reinserción laboral se consiguió en el $83,8 \%(119 /$ 142) de los pacientes tratados. Tras cinco años de seguimiento, se constata la consistencia del resultado clínico.

Discusión. Se observa el comportamiento del disco intervertebral en cuanto a las variables de sexo, edad, lateralidad, tipo de hernia, disco adicional, resultado a largo plazo y reintervenciones. $\mathrm{Al}$ analizar el tipo de hernia y la existencia de disco adicional, se han definido tres patrones de afección discal. Se considera a la microdiscectomía lumbar como la técnica de elección

Recibido: 17-09-10. Aceptado: 6-10-10 para tratar las recidivas discales.

Conclusiones. Entre la población laboralmente activa, la patología discal predomina en el varón joven, como consecuencia de una rotura del anillo, o una rotura del anillo y del ligamento longitudinal posterior (hernia traumática). Con frecuencia se observa una afectación de más de un disco y una lateralización izquierda.

PALABRAS CLAVE. Microdiscectomía lumbar. Hernia traumática. Medio laboral.

Long-term outcomes of lumbar microdiscectomy in a working class sample

\section{Summary}

Introduction. In the treatment of the lumbar disc herniaton (LDH) microdiscectomy constitutes one of the standard procedures. In the present study we have analyzed the clinical outcome of the lumbar microdiscectomy in a series of worker patients who underwent surgery in our service.

Methods. Retrospective analysis and a 5-year follow up, of a series of 142 patients operated on by means of lumbar microdiscectomy in the $2004-2005$ period. The clinical outcome was analyzed according to the "Herron and Turner" outline: pain reduction, use of medical treatment, restriction in the ability to perform physical activities, and return to work.

Results. 116 men and 26 women, with an average age of 37.9 and 45.4 years respectively, underwent surgery because of LDH. In the clinical aspect, sciatica was predominant over low back pain in a ratio of three to one. The L5-S1 discal level was operated on in $68.3 \%$ of the cases. It was considered that occupational activities gave rise to damage in 107 patients $(\mathbf{7 5 . 3 \%}$ ). Besides a symptomatic disc, there was an additional injured disc in $44.3 \%$ of the cases. An initial unfavourable outcome was seen in 42 patients $(33 \%), 15$ of which recovered from in an interval of 3 months, and another fifteen within a one year period. A re-operation was necessa- 
ry in 16 patients because of recurrent lumbar disc herniation (11\%). Work reintegration was achieved in 83.3\% (119/142) of the cases. After a 5-year follow up, we stated the consistency of the clinical result.

Discussion. We analyzed the intervertebral disc behaviour as regards sex, age, variety of discal herniation, additional disc, outcome and re-operation variables. After the analysis of the type of discal herniation and additional disc we defined three disc injury patterns. We consider microdiscectomy as the technique of choosing for the treatment of recurrence disc herniation.

Conclusions. Between the working class, discal injury predominates in young men, as a consequence of the annulus breakage, or an annulus plus posterior longitudinal ligament breakage (traumatic herniae). Frequently it was observed that more than one disc was involved, and a left lateralization.

KEY WORDS. Lumbar microdiscectomy. Traumatic herniae. Occupational activities.

\section{Introducción}

En el tratamiento de la hernia de disco lumbar (HDL), la microdiscectomía constituye hoy día un estándar entre las distintas opciones quirúrgicas existentes ${ }^{7,10-13,23,27}$. Un buen resultado se obtiene en el 76-93\% de los casos, según las series consultadas ${ }^{18,21,29}$. Se considera que los pacientes que han sido intervenidos por una HDL presentan un riesgo del 5 al $12.5 \%$ de volver a ser operados (nueva microdiscectomía u otro tipo de cirugía descompresiva o estabilizadora) en los siguientes 20 años $^{19,24}$. El porcentaje global de reintervenciones por una HDL recurrente, según las series consultadas, es del 3 al 11\% $0^{5,9,25,28}$.

La finalidad del presente estudio ha sido analizar el resultado clínico de una serie de pacientes, laboralmente activos, intervenidos mediante microdiscectomía, en un hospital con especial dedicación al accidente de trabajo, buscando definir las variables que discriminen el origen traumático de la enfermedad discal.

\section{Material y métodos}

Diseño del estudio: análisis retrospectivo y seguimiento de 5 años del resultado de la microdiscectomía en un grupo de pacientes intervenidos por hernia discal lumbar en el Servicio de Neurocirugía del Hospital MAZ en los años 2004 y 2005.

Se incluyeron en este estudio los pacientes afectos de un cuadro clínico de lumbalgia y/o ciatalgia rebelde a tratamiento médico convencional: tratamiento médico analgésico/antiinflamatorio combinado con terapia física/
$2011 ; 22: 235-244$

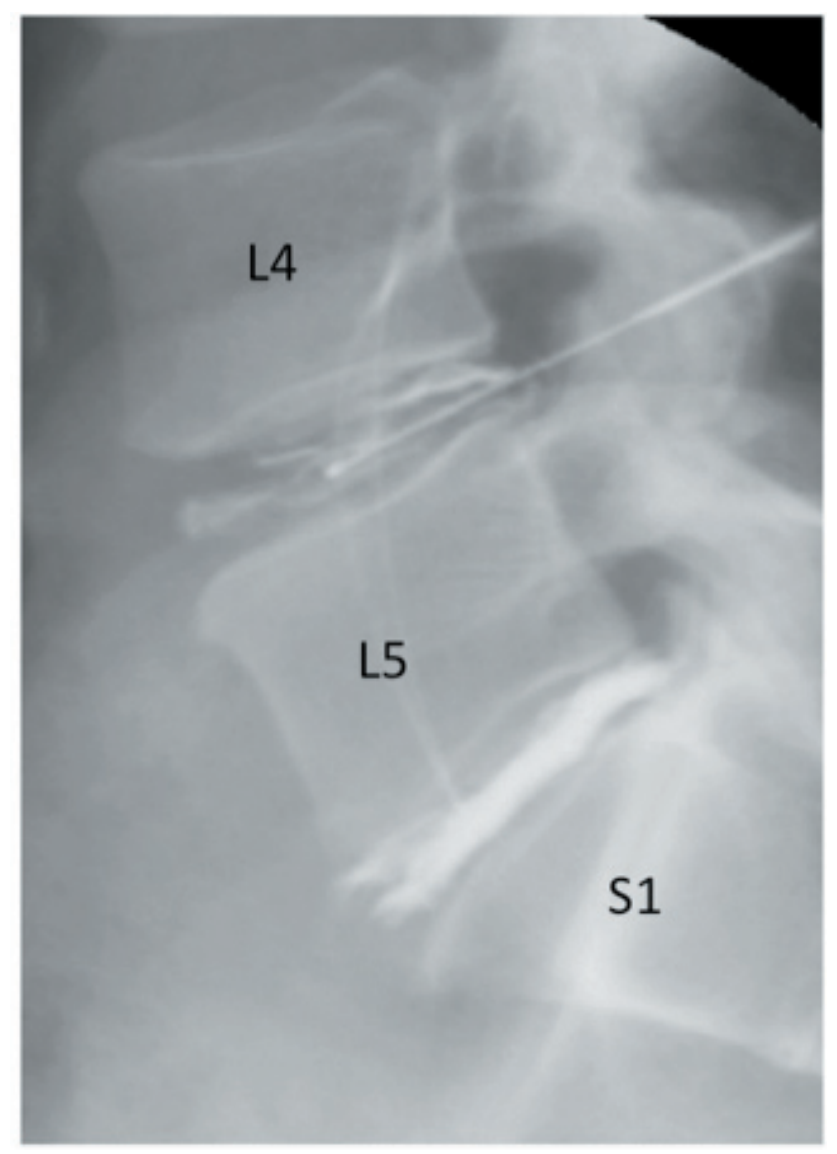

Figura 1. Imagen que muestra discografia L4-L5 (concordante) y L5-S1 (no doloroso). Se corresponde con la imagen de RM del disco tipo I de la Tabla I.

rehabilitadora, que se prolongó una media de 2,3 meses $(1,2-3,1)$. La confirmación de la sospecha clínica se estableció mediante estudio de resonancia magnética -RM- (Philips-Intera 1T-Holanda; proyecciones sagitales TSE/T1 y TSE/T2, y transversales TSE/T2). En aquellas situaciones en las que apreciamos más de un disco dañado y dudas razonables sobre cuál de ellos era el causante de la clínica del paciente, se practicó una discografía de los discos problema (Figura 1). Se han descartado del estudio aquellos pacientes con más de un disco sintomático.

En todos los casos intervenidos se registró: si predominaba la lumbalgia o la ciatalgia, si el origen del proceso se encontraba relacionado con un accidente de trabajo (categoría laboral) o no (categoría no laboral), el nivel discal afectado, la existencia de patología discal asociada, el tipo de hernia discal según el hallazgo quirúrgico (tipos I, II y III -según protocolo seguido en nuestro Centro-), las complicaciones y, el resultado clínico a corto (tres meses) y a largo plazo (1 y 5 años).

Nuestro procedimiento microquirúrgico lo realizamos a través de un abordaje mínimamente invasivo previo mar- 


\section{Tabla 1}

Clasificación tipos de hernias. Las imágenes han sido elegidas tras definir el tipo de hernia durante el acto quirúrgico

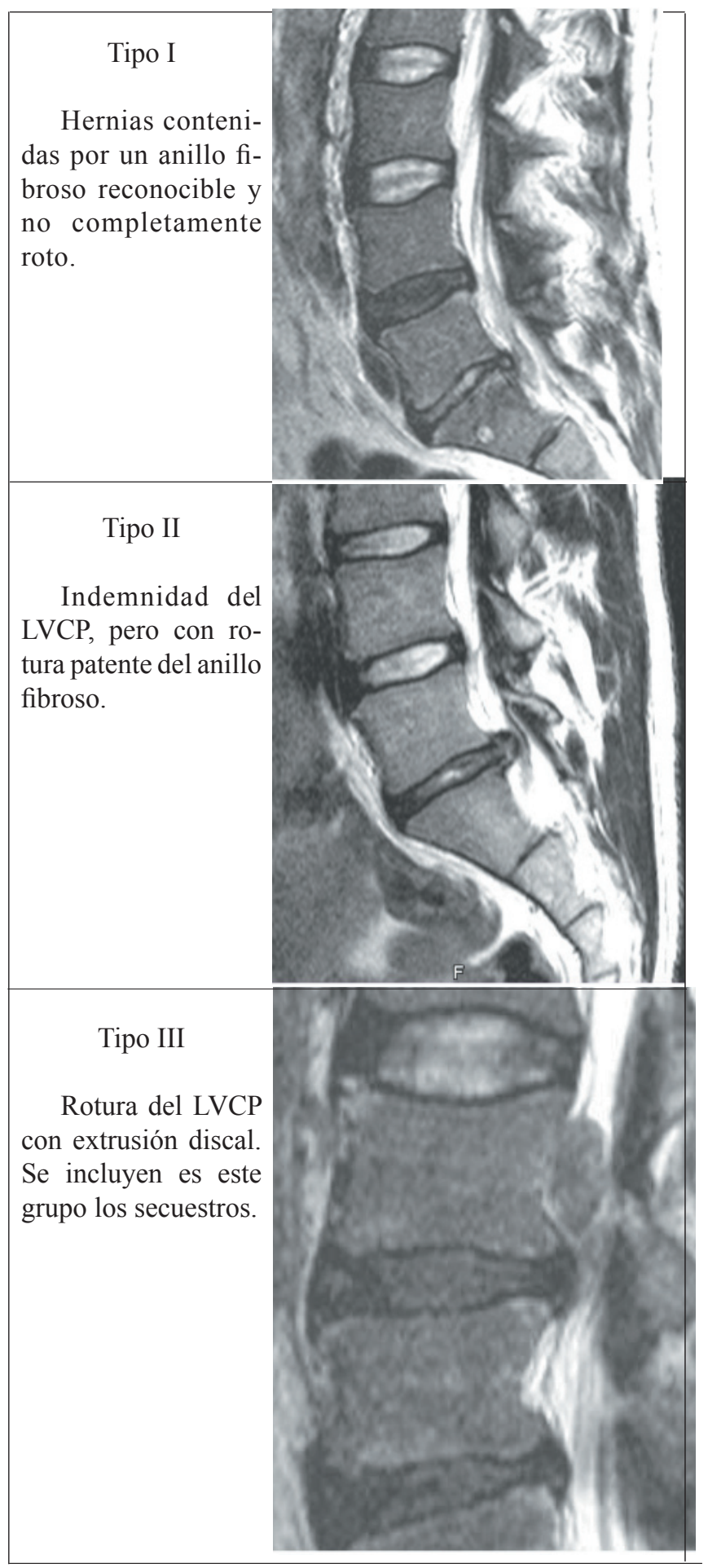

cado radiológico, utilizando un sistema retractor expansible Spine Classics HLD (Aesculap); la técnica no se limita a la resección de los fragmentos extruídos o secuestros, sino que se completa con una discectomía agresiva aunque sin curetaje de los platillos vertebrales.

Muchas veces con una imagen de RM es difícil establecer el grado de extrusión del núcleo pulposo, sobre todo a la hora de definir la rotura o no del ligamento vertebral común posterior (LVCP), es por ello que la catalogación del tipo de hernia la hemos realizado durante el acto microquirúrgico, puesto que durante el mismo podemos apreciar, bien la integridad del LVCP (su integridad descartaría la hernia de tipo III), bien la integridad del anillo fibroso (su integridad descartaría la hernia de tipo II) . Tabla 1.

El resultado clínico fue evaluado en el $100 \%$ de los casos según el esquema propuesto por Herron y Turner en base al uso de analgésicos, restricción de actividades físicas (de ninguna a grave) y estado laboral (regreso al trabajo previo, reubicación laboral o incapacidad laboral $)^{16,17}$. Se sobreentiende que un paciente que regresa a su trabajo previo, no presenta restricciones en las actividades físicas (o son mínimas) y no precisa de analgesia habitual.

En nuestro Centro hemos definido que un paciente sufre una recidiva discal, cuando en las imágenes de RM se aprecia la herniación en el mismo nivel que el intervenido, con independencia de su lateralidad, y con un intervalo libre de síntomas superior a 6 meses. Por debajo de este período consideramos que la clínica es secundaria a una compresión radicular residual. En los pacientes sometidos a reintervención se analizó la manera de incidir el anillo fibroso (bien en cruz, bien en cuadrado) y, la altura discal tras la primera cirugía, por si pudieran influir en la aparición de recidivas.

Los datos han sido tratados mediante análisis estadístico con el fin de determinar qué variables discriminarían entre el origen traumático o no traumático de la enfermedad discal, y cuáles son las características de las hernias discales lumbares producidas en accidentes laborales, utilizando para ello el programa de libre distribución G-Stat 2.0 (htpp://www.e-biometria.com/g-stat/index.html).

En este manuscrito hemos utilizado los conceptos de "disco sintomático" para definir el que origina la clínica del paciente; "disco adicional afecto" para definir el disco intervertebral dañado, pero sin relevancia clínica; "accidente laboral" para definir toda lesión que acontece o es consecuencia del trabajo, incluidos los desplazamientos relacionados con el mismo.

\section{Resultados}

Durante el bienio 2004-2005 fueron intervenidos por hernia discal lumbar 142 pacientes, 116 hombres (81.6\%) y 26 mujeres (18.3\%), con una edad media de 40.8 años (rango de 22 a 69). La edad media de los varones fue de 37.9 años, y de las mujeres 45.4 ( $\mathrm{p}=0.008)$. El porcen- 
Tabla 2

Distribución por nivel intervenido y clínica predominante

\begin{tabular}{|l|c|c|}
\hline $\begin{array}{l}\text { Nivel } \\
\text { intervenido }\end{array}$ & $\begin{array}{c}\text { Lumbalgia } \\
\mathrm{n}^{\mathrm{o}}(\%)\end{array}$ & $\begin{array}{c}\text { Citalgia/cruralgia* } \\
\mathrm{n}^{\circ}(\%)\end{array}$ \\
\hline L5-S1 & $24(16.8)$ & $73(51.4)$ \\
\hline L4-L5 & $9(6.3)$ & $28(19.7)$ \\
\hline L3-L4 & $4(2.8)$ & $2(1.4)^{*}$ \\
\hline Otros & $2(1.4)$ & - \\
\hline Totales & $39(27.4)$ & $103(72.5)$ \\
\hline
\end{tabular}

taje de seguimiento de los pacientes fue del $100 \%$. No se registró ningún síndrome de cola de caballo. Se observó un claro predominio de la ciatalgia sobre la lumbalgia en una proporción de 3 a 1 (Tabla 2). En 23 pacientes un cuadro deficitario motor fue predominante (déficits S1: 14 casos; L5: 6 casos; L4: 2 casos; L3: 1 caso). No apreciamos amiotrofias.

La distribución por disco intervenido figura en la Tabla 3. En el $68.3 \%$ de los casos el disco intervenido fue el L5S1, seguido de lejos del L4-L5. Como puede apreciarse, los pacientes con HD de nivel más alto, presentaban mayor edad. El contraste de medias de edad (ANOVA) resultó significativamente mayor en L5-S1 frente a L3-L4 $(\mathrm{p}<0,05)$.

Un hallazgo relevante en nuestra serie fue que en el $59,15 \%$ de los casos existía una lateralización hacia la izquierda de la hernia discal.

En la Tabla 4 podemos apreciar que en 107 casos se consideró el origen del padecimiento como accidente de trabajo (75.3\%), 94 de ellos varones. En nuestra serie encontramos una clara relación entre el sexo y el origen laboral o no del proceso. En el 66,2\% de los casos se asociaron las variables "sexo varón" y "origen laboral", mientras que en el 9,1\% lo hicieron "origen laboral" y "sexo femenino" ( $p<0,001)$. No se apreció relación entre el nivel herniado y el origen laboral o no del cuadro. Sin embargo, si consideramos el tipo de hernia, los accidentes laborales tienden a asociarse con los tipos II y III, en las que existe una rotura bien del anillo o del ligamento vertebral común posterior ( $\mathrm{p}=0.02)$ Gráfico 1 .

En el estudio de la RM, apreciamos que en el $44.3 \%$ de los pacientes (63/142), además del disco sintomático, existía un disco adicional afecto. Tabla 5. La proporción de casos con un disco adicional afecto fue menor entre los pacientes con un origen no laboral del proceso $(\mathrm{p}<0.01)$. Tabla 6.

En $47(33 \%)$ de los 142 casos, reconocimos una evolución postquirúrgica inicial desfavorable, secundaria a lumbalgia postquirúrgica en 22 pacientes, radiculitis

Tabla 3

Distribución por nivel intervenido y tipo de hernia discal

\begin{tabular}{|l|c|c|c|c|c|}
\hline $\begin{array}{c}\text { Nivel } \\
\text { intervenido }\end{array}$ & $\mathrm{N}^{\mathrm{o}}(\%)$ & $\begin{array}{c}\text { Edad media } \\
(\mathrm{años})\end{array}$ & $\begin{array}{c}\text { Tipo I } \\
\mathrm{n}^{\circ}(\%)\end{array}$ & $\begin{array}{c}\text { Tipo II } \\
\mathrm{n}^{\mathbf{o}}(\%)\end{array}$ & $\begin{array}{c}\text { Tipo III } \\
\mathrm{n}^{\mathbf{o}}(\%)\end{array}$ \\
\hline L5-S1 & $97(68.3)$ & 39.9 & $25(17.6)$ & $35(24.7)$ & $37(26.0)$ \\
\hline L4-L5 & $37(26)$ & 41.7 & $9(6.3)$ & $11(7.7)$ & $17(12.0)$ \\
\hline L3-L4 & $6(4.23)$ & 49.5 & - & $5(3.5)$ & $1(0.7)$ \\
\hline Otros & $2(1.4)$ & 35.0 & - & $1(0.7)$ & $1(0.7)$ \\
\hline Totales & $142(100)$ & 40.8 & $34(23.94)$ & $52(36.62)$ & $56(39.44)$ \\
\hline
\end{tabular}

Tabla 4

Tabla de frecuencias de sexo por laboral/no laboral

\begin{tabular}{|l|c|c|c|}
\hline & Laboral n / (\%) & No laboral n / (\%) & Totales n / (\%) \\
\hline Hombre & $94 / 66.2$ & $22 / 15.49$ & $116 / 81.69$ \\
\hline Mujer & $13 / 9.15$ & $13 / 9.15$ & $26 / 18.31$ \\
\hline Totales & $107 / 75.35$ & $35 / 24.65$ & $142 / 100.00$ \\
\hline
\end{tabular}




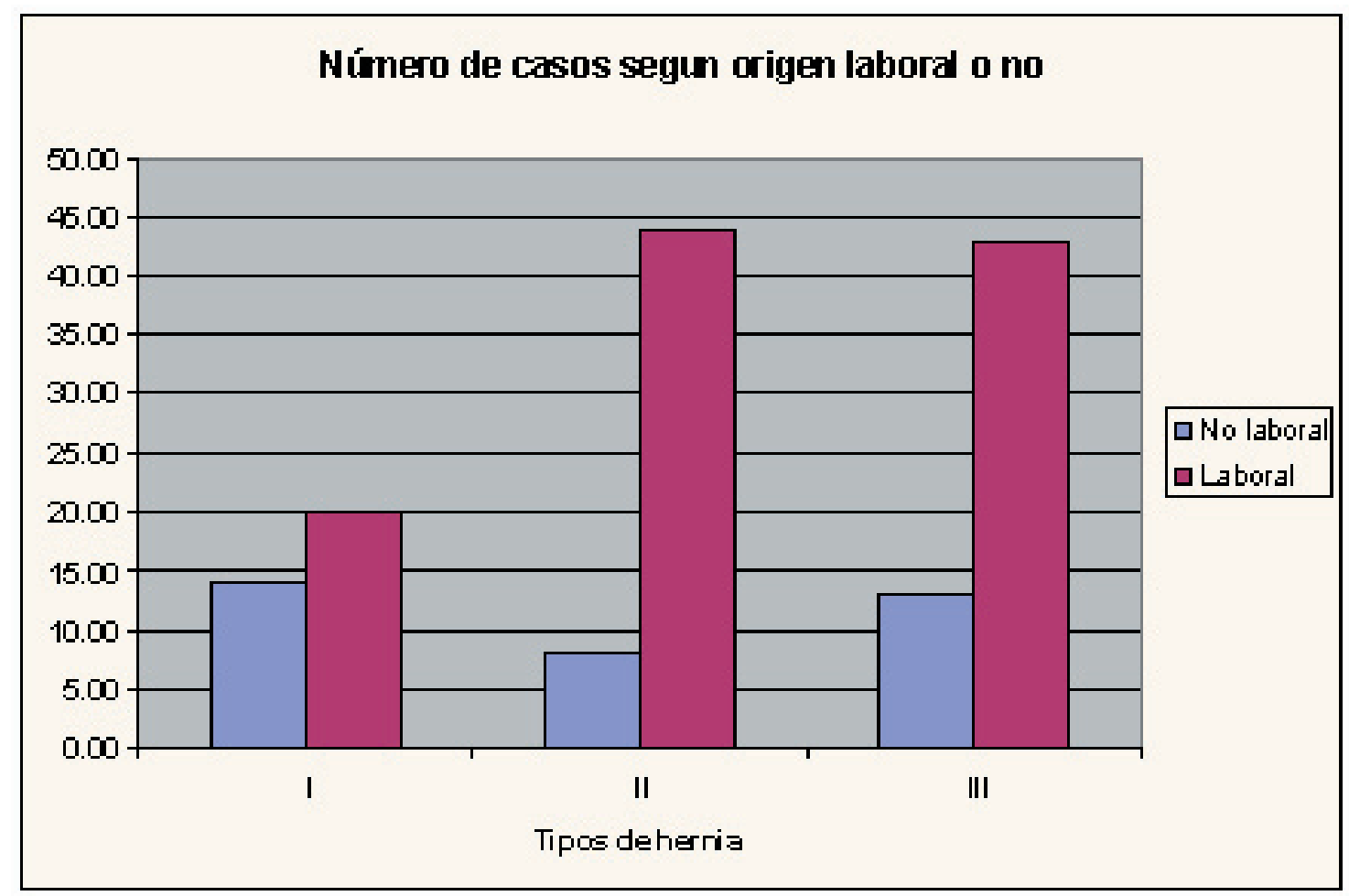

Gráfico 1. Relación tipo de hernia y categorización laboral/no laboral

Tabla 5

Distribución discos sintomático/adicional

\begin{tabular}{|cc|c|c|c|c|}
\hline \multicolumn{2}{|c|}{$\begin{array}{c}\text { (Disco sintomático) } \\
\text { / Disco adicional }\end{array}$} & L5-S1 & L4-L5 & L3-L4 & Otro nivel / Más de uno \\
\hline L5-S1 & $(97) / \mathbf{3 9}$ & 0 & 24 & 3 & $3 * / 9$ \\
\hline L4-L5 & $(37) / \mathbf{1 9}$ & 9 & 0 & 1 & $2 * * / 7$ \\
\hline L3-L4 & $(6) / \mathbf{5}$ & 2 & 2 & 0 & $1 * * * /-$ \\
\hline & Totales & 11 & 26 & 4 & $6 / 16$ \\
\hline
\end{tabular}

*L2-L3: 1 caso; D11-D12: 2 casos /**L2-L3: 2 casos /***D11-D12: 1 caso

postoperatoria en once y de debilidad motora persistente en tres. En ocho casos se desarrollaron seromas; tres pacientes sufrieron flebotrombosis ipsilateral a la lateralización de la hernia discal. Tras tratamiento médico y rehabilitador se consiguió recuperar en un plazo menor de 3 meses, a 15 de los 47 pacientes anteriormente comentados. En el resto (32/47) persistió la lumbalgia postquirúrgica (20 casos), las sensaciones disestésicas ipsilaterales a la lateralización discal -radiculitis- (10 casos), y la debilidad motora (2 casos), lo que obligó a mantener en estos pacientes, la restricción de actividades físicas y la utilización de un tratamiento médico ad hoc, evitando, por política de nues-
Tabla 6

Distribución de disco adicional con respecto a si el paciente es de índole laboral o no

\begin{tabular}{|c|r|c|c|}
\hline Disco adicional & Sí & No & Totales \\
\hline Laboral & 54 & 53 & 107 \\
\hline No laboral & 9 & 26 & 35 \\
\hline Totales & 63 & 79 & 142 \\
\hline
\end{tabular}

tro Servicio, el uso de narcóticos. De estos 32 pacientes (22,5\% del total), se consiguió a lo largo de un año reubicar 
Tabla 7

Seguimiento de los pacientes con mala respuesta terapéutica

\begin{tabular}{|l|c|c|c|}
\hline & Postquirúrgico & 3 meses & 1 año \\
\hline Lumbalgia & 22 & 20 & $11^{*}$ \\
\hline Radiculitis & 11 & 10 & $4 * *$ \\
\hline Debilidad motora & 3 & 2 & 2 \\
\hline Infección superficial & 8 & - & - \\
\hline Flebotrombosis & 3 & - & - \\
\hline \multicolumn{1}{|c|}{ Totales } & $\mathbf{4 7}$ & $\mathbf{3 2}$ & $\mathbf{1 7}^{\#}$ \\
\hline
\end{tabular}

* Reintervención quirúrgica en 2 casos: fijación transpedicular

** Reintervención quirúrgica en 1 caso: fijación transpedicular \# No reinserción laboral

Tabla 8

Tabla de frecuencias de disco adicional (filas) y evolución desfavorable (columnas)

\begin{tabular}{|c|c|c|c|}
\hline $\begin{array}{l}\text { Evolución } \\
\text { desfavorable } \\
\text { Disco adicional }\end{array}$ & No & Sí & Totales \\
\hline No & $60(42.25)$ & $19(13.387)$ & $79(55.63)$ \\
\hline Sí & $35(24.65)$ & $28(19.72)$ & $63(44.37)$ \\
\hline Totales & $95(66.9)$ & $47(33.1)$ & $142(100.0)$ \\
\hline
\end{tabular}

Tabla 9

Tabla de frecuencias de reinserción laboral (filas) y origen del proceso (columnas)

\begin{tabular}{|l|c|c|c|}
\hline $\begin{array}{c}\text { Reinserción laboral } \\
\text { Origen del proceso }\end{array}$ & $\begin{array}{c}\text { S í } \\
\mathrm{n}^{\mathbf{0}} /(\%)\end{array}$ & $\begin{array}{c}\text { No } \\
\mathrm{n}^{\mathrm{o}}(\mathbf{\%})\end{array}$ & $\begin{array}{c}\text { Totales } \\
\mathrm{n}^{\mathbf{0}} /(\%)\end{array}$ \\
\hline No laboral & $33(23.4)$ & $2(1.41)$ & $35(24.65)$ \\
\hline Laboral & $86(60.56)$ & $21(14.79)$ & $107(75.35)$ \\
\hline \multicolumn{1}{|c|}{ Totales } & $119(83.8)$ & $23(16.2)$ & $142(100)$ \\
\hline
\end{tabular}

laboralmente a quince, siendo remitidos el resto (11.9\%) a Tribunal Médico por incapacidad laboral. Tabla 7.

Estimamos, por otro lado, que los pacientes con disco adicional afecto tuvieron una mayor tendencia a sufrir una evolución desfavorable con respecto a los que no lo presentaron $(\mathrm{p}<0.01)$ Tabla 8.

Consideramos necesaria la reintervención quirúrgica en 16 pacientes por recidiva discal ( $11.2 \%$ del total). Con excepción de tres casos reintervenidos antes de completar el primer año de seguimiento, los otros trece pacientes se habían reintegrado a su vida laboral habitual. Los nuevos estudios de RM determinaron que en once de los 16 pacien- tes existía un disco adicional afecto. Salvo en cuatro casos, en los que se consideró necesaria una fijación transpedicular (signos radiológicos de inestabilidad), en los demás se practicó una nueva microdiscectomía sobre el mismo nivel intervenido, apreciándose en todos ellos fragmentos discales extruídos. Las recidivas mantuvieron la misma lateralidad que en el episodio previo. En 9 pacientes el nivel afecto fue el L5-S1, mientras que en 7 se afectó el L4-L5. El tiempo medio entre las cirugías fue de 18 meses (rango de 7 a 23 ). Se midió el espacio intervertebral del segmento problema en los pacientes que fueron reintervenidos, de forma previa a cada una de las cirugías, comprobándose que existía una disminución media de la altura discal de $0.5 \mathrm{~mm}(0-1,6)$. Comprobamos que los pacientes reintervenidos eran 5 años de media más jóvenes que el resto de la serie (diferencia no significativa). En dos de los pacientes sometidos a una segunda microdiscectomía, fue preciso realizar una tercera. La re-recidiva se instauró a los 11 meses y 14 meses, respectivamente, de la segunda intervención. Ambos pacientes habían sido dados de alta laboral y se habían reintegrado a su trabajo habitual. La clínica resultó similar a las situaciones previas. En los dos casos pudo practicarse una nueva microdiscectomía sin incidencias, completándose la técnica con un espaciador interespinoso.

Al comparar la edad media de los pacientes que aquejaban lumbalgia postquirúrgica (44.6 años), con la de los que se sometieron a reintervención mediante microcirugía (36 años), apreciamos una diferencia estadísticamente significativa (ANOVA: $\mathrm{p}<0,05$ ). No encontramos asociación entre la aparición de complicaciones o la necesidad de reintervención quirúrgica, y el origen laboral o no del proceso.

Tras 5 años de seguimiento, la reinserción laboral se consiguió en $119(83.8 \%)$ de los 142 pacientes tratados: de los 12 pacientes sometidos a una reintervención mediante microdiscectomía, se consiguió, en principio, la reinserción laboral en nueve. A los dos pacientes posteriormente reintervenidos, se les concedió una incapacidad para su trabajo habitual. Los 4 pacientes reintervenidos que precisaron de una fijación transpedicular, no se reincorporaron a la vida laboral ( 3 de ellos reintervenidos en el primer año de evolución). En total, tras el primer año de seguimiento, 6 pacientes más perdieron la capacitación para su trabajo habitual. Hemos apreciado una mayor tendencia a favor de la reinserción laboral cuando el origen del proceso no es laboral, pero este dato se halla ligeramente por encima de la significación estadística $(\mathrm{p}=0.05)$. Tabla 9 .

Así mismo, los pacientes con un disco adicional afecto se reincorporan menos a su trabajo al compararlos con los que no lo padecían $(39.50 \%$ frente a $60.50 \%$; $<<0.001)$.

Por otro lado, los pacientes que sufren complicaciones a largo plazo, se reincorporan menos a la vida laboral $(51 \%$ de reincorporaciones), al compararlo con los que no las 
padecen $(98.9 \%$ de reincorporaciones) ( $\mathrm{p}<0.001)$. A los cinco años de seguimiento, es importante destacar la consistencia del resultado clínico, con independencia de que hayan podido ser asistidos por lumbociatalgias ocasionales, lográndose cerrar el episodio que motivara su asistencia en cerca del $84 \%$ de los pacientes tratados.

No hemos encontrado en nuestra serie, diferencias significativas en razón del sexo, por disco herniado, lateralidad, tipo de hernia, complicaciones o reintervenciones. La lateralidad no influye en la respuesta al tratamiento quirúrgico, ni en las complicaciones ni en la reincorporación laboral.

\section{Discusión}

Basta con revisar la literatura médica para constatar que el resultado que se obtiene tras la discectomía lumbar es contradictorio. Existen revisiones que establecen que hasta el $40 \%$ de los pacientes sometidos a cirugía discal lumbar continúan padeciendo ciática recurrente o persistente, dolor crónico de espalda o recidiva discal ${ }^{3}$. Es más, el resultado quirúrgico tiende a ser peor en aquellos pacientes que esperan una indemnización laboral ${ }^{15,20}$.

Ahora bien, si existen trabajos que analizan cómo influye la litigación en el resultado de cirugía discal lumbar, son pocos los trabajos que estudian la forma en que se daña el disco intervertebral (DI) en el medio laboral. En nuestra serie, se constató el origen laboral del padecimiento en las tres cuartas partes, lo que nos ha permitido analizar el comportamiento del DI en una población laboralmente activa:

\section{Sexo}

El primer dato a considerar es la diferencia de sexo observada en nuestra muestra, ofreciendo una clara incidencia mayor en los hombres. La explicación más probable es que se trata de una patología de esfuerzo, procedente, además, en su mayoría de accidentes laborales. La población masculina está más expuesta a trabajos que implican cargas de raquis y esto podría explicar que las mujeres, mayoritariamente dedicadas a trabajos tipo white collar, experimenten esta patología en una clara menor medida. Creemos que sólo las razones mecánicas explican este hecho, ya que otras variables como la elasticidad ligamentaria, el menor elemento de contención articular a cargo de la masa muscular, etc, obrarían en el sentido contrario, es decir aumentando los casos en la población femenina, lo que no ocurre aquí. El hecho de que nuestra muestra incluya mayoritariamente accidentes laborales apoya esta hipótesis, ya que sólo en el 9,1\% del total de casos se asoció el sexo femenino con el origen laboral del padecimiento.

\section{Edad}

Respecto a la edad, en general, es una patología de población joven. Resulta interesante la diferencia de edad observada también entre hombres y mujeres, con tendencia a una menor edad media en estas últimas. Es posible que la población femenina más joven se halle más expuesta a factores mecánicos, quizá por la asunción de roles cada vez más demandantes físicamente para el sexo femenino en etapas más tempranas, bien por la acción de elementos de contención que protegen al hombre desde una edad menor (musculación). También parece que la edad se asocia al nivel de disco herniado. Así, los niveles altos se dan más en personas con mayores medias de edad. Probablemente, ello sea debido a razones biomecánicas, o a estados previos de la columna. Así, es posible que los factores mecánicos actúen en los jóvenes más sobre áreas expuestas a sobreesfuerzos, pero preserven relativamente otras regiones que como los discos altos reciben menor carga. La edad, al degenerar también áreas menos expuestas a microtraumatismos como los discos altos situaría a éstos en un estado de mayor vulnerabilidad a las cargas o sobreesfuerzos aumentando la incidencia de hernias en espacios superiores al L4-L5.

\section{Lateralidad}

Una hallazgo sorprendente y no reflejado, hasta donde nosotros sabemos, por la literatura es la clara predilección de las hernias por el lado izquierdo. En nuestro estudio, no hemos encontrado ninguna asociación de la lateralidad con ninguna otra variable de las analizadas. Únicamente podemos aventurar por ello razones biomecánicas para explicar esta diferencia. Probablemente, la población mayoritariamente diestra tenga tendencia a herniarse por el lado izquierdo debido a una mayor apertura homolateral del disco coincidiendo con compresión derecha (lado dominante) del núcleo pulposo en diestros. Este hecho requiere no obstante confirmación a través de estudios dirigidos a investigar este hallazgo.

\section{Tipo de hernia}

La clasificación usada por nosotros se halla muy orientada hacia los factores biomecánicos relacionados con el origen de la hernia discal. De hecho, los tipos II y III indican serias roturas discales, expresión frecuente de esfuerzos graves o sobrecargas agudas recayendo sobre el disco intervertebral. Ello explicaría que estos dos tipos sean los mas frecuentemente encontrados entre los accidentes laborales, tal y como indican nuestros resultados. Dicho de otro modo, estos dos tipos de hernia de disco podrían ser utilizados como marcadores de un origen traumático de una hernia, lo que puede tener interés en Medicina Laboral o implicaciones medico-legales, ya que a veces se discute sobre el origen traumático o degenerativo de las hernias de disco.

Deseamos reseñar que no ha sido la intención de este 
trabajo realizar una correspondencia de los hallazgos operatorios con las imágenes radiológicas. Sí hemos constatado sin embargo, que en muchas ocasiones la rotura del LVCP es difícil de determinar en la RM.

\section{Afectación de disco adicional}

Resulta interesante considerar el significado del hallazgo de RM de discos afectos, en mayor o menor medida, en la vecindad del disco herniado sintomático. La menor frecuencia de dicha afectación adicional en los casos no laborales podría también indicar que en los sobreesfuerzos o sobrecargas agudas que dan origen a una hernia de disco sintomática, no sólo se daña el disco principalmente afecto radiológicamente sino que pueden comprobarse alteraciones en discos adicionales. Lógicamente, no podemos excluir tampoco que la degeneración discal en tales casos sea preexistente y que el paciente herniado agudamente en un accidente laboral pueda portar ya degeneraciones previas en los discos. Pero resulta llamativo que la mayor parte de nuestra muestra de accidentes laborales sean personas sanas en cuyos reconocimientos de empresa no se advierten en general problemas adicionales de la espalda. Creemos por tanto que la explicación más plausible es que en el accidente no sólo pueda lesionarse un disco sino más de uno, un concepto pocas veces subrayado. Quizá el hecho de que se suele prestar más atención al disco afecto primariamente, influye en dedicar poco esfuerzo al tratamiento de los otros discos potencialmente dañados, lo que explicaría el peor pronostico observado en los pacientes con afectación discal doble o múltiple, tal y como pone de manifiesto nuestro trabajo. Así, en la mitad de los pacientes con una hernia discal asociada al trabajo, existía un disco adicional afecto. Esto nos ha llevado a plantearnos distintos patrones, con finalidad inicial descriptiva:

PRIMER PATRÓN. Disco sintomático: L5-S1; disco adicional: L4-L5.

SEGUNDO PATRÓN. Disco sintomático: L4-L5; disco adicional: L5-S1.

TERCER PATRÓN. Disco sintomático: L3-L4; disco adicional: L4-L5 ó el L5-S1.

Habría que llevar a cabo un estudio prospectivo para definir su utilidad terapéutica y/o pronóstica.

\section{Resultado a largo plazo}

Para Loupasis et al., el resultado a largo plazo de la discectomía lumbar estandarizada no es muy satisfactorio, ya que en un tercio de los pacientes el resultado es pobre $\mathrm{y}$ en una cuarta parte persiste un dolor residual significativo $^{21}$. Para otros, el uso del microscopio no modifica el resultado quirúrgico a largo plazo, debiendo dejar el uso del mismo al hábito del cirujano ${ }^{26}$. Es más, el abordaje lumbar mínimamente invasivo y microquirúrgico ha sido utilizado por otros grupos sin que éste influya negativamente en el resultado de la cirugía ${ }^{14}$.

En un reciente estudio clínico realizado por DeBerard MS et al., sobre qué variables prequirúrgicas se correlacionarían con un mal resultado, se establece como predictibles de mal pronóstico la edad (el paciente con más años dispondría de menor número de recursos biofísicos sanadores), la morbidez (fundamentalmente la depresión asociada -que intervendría en el mantenimiento y la exacerbación del dolor crónico-), la litigación (variable que claramente empeora el resultado clínico) y el tiempo de espera para la cirugía (a mayor espera, mayor pérdida de la condición física, o desarrollo de estados clínicos distintos) ${ }^{6}$.

Otros autores, han constatado que un estado de ansiedad prequirúrgico constituye un factor de mal pronóstico a largo plazo ${ }^{11}$. En este sentido, nuestro grupo ha establecido que la excesiva preocupación sobre el estado físico tras la cirugía influiría claramente hacia un mal pronóstico ${ }^{2}$.

Aunque para Moore AJ et al. no existen factores predictibles fiables a largo plazo ${ }^{23}$, nosotros hemos apreciado que un buen indicador a largo plazo de un resultado positivo lo constituye una situación clínica favorable de forma precoz. Distintos estudios corroboran este aserto, con independencia del índice o índices utilizados para dicha medida (escala visual analógica, índice de incapacidad de Oswestry, índice de incapacidad de Million; clasificación de Macnab, cuestionario de Roland-Morris, etc.) $)^{7,10,12,13,23}$. Es más, hemos apreciado que si tras seis meses de convalecencia postquirúrgica un paciente no puede ser dado de alta laboral, es difícil que éste vuelva a trabajar. Hakkinen et al., también apreciaron que si el estado clínico del paciente es bueno al segundo mes de la cirugía la probabilidad de que lo sea al final del año es elevada ${ }^{13}$. Y en este sentido, Findlay et al. corroboran que si la situación clínica a los 6 meses fuera correcta, a los 10 años tiende a mantenerse ${ }^{10}$. Otros autores han encontrado que el resultado quirúrgico era mejor en los pacientes en los que predominaba la ciatalgia sobre la lumbalgia, en los pacientes con secuestro discal al compararlas con las hernias extruídas o contenidas $^{7}$. Para ellos, las HD L5-S1 evolucionaban mejor que las L4-L57.

\section{Reintervenciones}

Keskimäki et al. constataron, tras analizar 25.359 casos del registro hospitalario finlandés de pacientes dados de alta médica, intervenidos por hernia discal lumbar en un periodo de 9 años, que el servicio con mayor numero de discectomías realizadas era el que mayor número de reintervenciones practicaba ${ }^{19}$.

La tasa acumulada de reintervenciones para Häkkinen et al. fue del $10 \%$ a los 5 años de seguimiento ${ }^{12}$, para Atlas et al. del $25 \%$ a los 10 años $^{1} \mathrm{y}$, para Osterman del $7 \%$ en el primer año y del $20 \%$ tras 10 años de seguimiento ${ }^{24}$. En nuestro estudio reintervenimos, a lo largo de los 5 años de 
seguimiento, al $11 \%$ de los pacientes.

En nuestra muestra apreciamos que el nivel y la lateralidad de los 12 pacientes reintervenidos por recidiva discal fue el mismo. Connolly apreció en su serie, que tras una discectomía L4-L5, la recurrencia predominaba en el mismo nivel (66\%), mientras que en los discos L5-S1 la recurrencia discal se producía por igual en el disco previamente intervenido como en el suprayacente ${ }^{5}$.

Otros autores han barajado como hipótesis de recidiva discal, el tipo de agresión que el anillo discal sufría durante la cirugía inicial ${ }^{28}$, el grado de degeneración discal ${ }^{4,8}$, o que se preserve la altura discal tras la cirugía del disco ${ }^{29}$. Al analizar estos aspectos en nuestra muestra comprobamos que el tipo de agresión quirúrgica sobre el anillo -incisión en cruz o en cuadrado- no influyó en la recidiva discal; el grado de degeneración discal era leve en nuestros pacientes $\mathrm{y}$, en los casos en los que se reintervino, prácticamente no se modificó la altura discal.

Al igual que Suk et al., creemos que la microdiscectomía es la técnica de elección para resolver una recurrencia discal $^{28}$. Que se complete la misma con una estabilización dependerá, en nuestra opinión, del grado de inestabilidad que se haya desarrollado en el segmento problema.

Algunos autores, tras una revisión sistematizada de la literatura médica, han sugerido que la microdiscectomía conservadora, al compararla con la agresiva, puede ofrecer un tiempo quirúrgico menor, una mayor prontitud en la reincorporación laboral, una menor incidencia de lumbalgia recurrente a largo plazo, pero un incremento en la incidencia de recidivas discales ${ }^{22,30}$. En nuestro estudio no hemos podido corroborar estos datos. El grupo de Fandiño et al. concluyó, tras analizar un grupo de 1.850 pacientes intervenidos por hernia discal lumbar, que los pacientes afectos de fibrosis postquirúrgica sintomática, no deben ser reintervenidos, puesto que sólo un 38\% de los reintervenidos por fibrosis alcanzó un buen resultado 9 . En nuestra serie no hemos llevado a cabo reintervenciones por fibrosis postquirúrgica.

\section{Conclusiones}

En la población laboralmente activa, la hernia discal tributaria de cirugía es una patología más frecuente en el varón, y con una predilección, en su lateralidad, hacia la izquierda, probablemente por razones biomecánicas. Con frecuencia se observa afectación de más de un disco. La hernia discal en el grupo femenino tendió a darse en edades más tempranas.

Los tipos de hernia que implican rotura bien del anillo, bien del anillo y del ligamento longitudinal posterior, son más frecuentes en accidentes laborales.

Podrían perfilarse como marcadores del origen laboral de una hernia discal:
- Sexo: varón

- Edad: sujeto joven (más en la mujer)

- Tipo de hernia: II y III

Otras variables que requerirían de más estudios al respecto:

- lateralización izquierda

- afección de más de un disco

\section{Bibliografía}

1. Atlas, S.J., Keller, R.B., Wu, Y.A., Deyo, R.A., Singer, D.E.: Long-term outcomes of surgical and nonsurgical management of lumbar spinal stenosis: 8 to 10 year results from the maine lumbar spine study. Spine (Phila Pa 1976) 2005; 30: 936-943.

2. Aso, J., Martínez Quiñones, J.V., Arregui, R.: Simulación en patología espinal. Eds. Grupo 2 Comunicación Médica SL. Madrid, 2005. (DL: M-4.785-2005).

3. Carragee, E.J., Han, M.Y., Suen, P.W., Kim, D.: Clinical outcomes after lumbar discectomy for sciatica: the effects of fragment type and anular competence. J Bone Joint Surg Am 2003; 85-A: 102-108.

4. Cinotti, G., Roysam, G.S., Eisenstein, S.M., Postacchini, F.: Ipsilateral recurrent lumbar disc herniation. A prospective, controlled study. J Bone Joint Surg Br 1998; 80: 825-832.

5. Connolly, E.S.: Surgery for recurrent lumbar disc herniation. Clin Neurosurg 1992; 39: 211-216.

6. DeBerard, M.S., LaCaille, R.A., Spielmans, G., Colledge, A., Parlin, M.A. Outcomes and presurgery correlates of lumbar discectomy in Utah Workers' Compensation patients. Spine J 2009; 9: 193-203.

7. Dewing, C.B., Provencher, M.T., Riffenburgh, R.H., Kerr, S., Manos, R.E.: The outcomes of lumbar microdiscectomy in a young, active population: correlation by herniation type and level. Spine 2008; 33: 33-38.

8. Dora, C., Schmid, M.R., Elfering, A., Zanetti, M., Hodler, J., Boos, N.: Lumbar disk herniation: do MR imaging findings predict recurrence after surgical diskectomy? Radiology 2005; 235: 562-567.

9. Fandiño, J., Botana, C., Viladrich, A., Gomez-Bueno, J.: Reoperation after lumbar disc surgery: results in 130 cases. Acta Neurochir (Wien) 1993; 122: 102-104.

10. Findlay, G.F., Hall, B.I., Musa, B.S., Oliveira, M.D., Fear, S.C.: A 10-year follow-up of the outcome of lumbar microdiscectomy. Spine 1998; 23: 1168-1171.

11. Graver, V., Haaland, A.K., Magnaes, B., Loeb, M.: Seven-year clinical follow-up after lumbar disc surgery: results and predictors of outcome.Br J Neurosurg 1999; 13: 178-184.

12. Häkkinen, A., Kautiainen, H., Järvenpää, S., ArkelaKautiainen, M., Ylinen, J.: Changes in the total Oswestry Index and its ten items in females and males pre- and post-sur- 
gery for lumbar disc herniation: a 1-year follow-up. Eur Spine J 2007; 16: 347-352.

13. Hakkinen, A., Ylinen, J., Kautiainen, H., Airaksinen, O., Herno, A., Kiviranta, I.: Does the outcome 2 months after lumbar disc surgery predict the outcome 12 months later? Disabil Rehabil 2003; 25: 968-972.

14. Harrington, J.F., French, P.: Open versus minimally invasive lumbar microdiscectomy: comparison of operative times, length of hospital stay, narcotic use and complications. Minim Invasive Neurosurg 2008; 51: 30-35.

15. Harris, I., Mulford, J., Solomon, M., van Gelder, J.M., Young, J.: Association between compensation status and outcome after surgery: a meta-analysis. JAMA 2005; 293: 16441652.

16. Herron, L.D., Turner, J.A.: Patient selection for lumbar laminectomy and discectomy with a revised objective rating system. Clin Orthop Relat Res 1985; 199: 145-152

17. Herron, L.D., Turner, J.A., Novell, L.A., Kreif, S.L.: Patient selection for lumbar discectomy with a revised objective rating system. Clin Orthop Relat Res 1996; 325: 148-155

18. Jansson, K.A., Németh, G., Granath, F., Blomqvist, P.: Surgery for herniation of a lumbar disc in Sweden between 1987 and 1999. An analysis of 27,576 operations. J Bone Joint Surg Br 2004; 86: 841-847.

19. Keskimäki, I., Seitsalo, S., Osterman, H., Rissanen, P.: Reoperations after lumbar disc surgery: a population-based study of regional and interspecialty variations. Spine 2000; 25: 1500-1508.

20. Klekamp, J., McCarty, E., Spengler, D.M.: Results of elective lumbar discectomy for patients involved in the workers' compensation system. J Spinal Disord 1998; 11: 277 282.

21. Loupasis, G.A., Stamos, K., Katonis, P.G., Sapkas, G., Korres, D.S., Hartofilakidis, G.: Seven- to 20-year outcome of lumbar discectomy. Spine 1999; 24: 2313-2317.

22. McGirt, M.J., Ambrossi, G.L., Datoo, G., Sciubba, D.M., Witham, T.F., Wolinsky, J.P., Gokaslan, Z.L., Bydon, A.: Recurrent disc herniation and long-term back pain after primary lumbar discectomy: review of outcomes reported for limited versus aggressive disc removal. Neurosurgery 2009;
64: $338-344$.

23. Moore, A.J., Chilton, J.D., Uttley, D.: Long-term results of microlumbar discectomy. Br J Neurosurg 1994; 8: 319-326.

24. Osterman, H., Sund, R., Seitsalo, S., Keskimäki, I.: Risk of multiple reoperations after lumbar discectomy: a population-based study. Spine 2003; 28: 621-627.

25. O'Sullivan, M.G., Connolly, A.E., Buckley, T.F.: Recurrent lumbar disc protrusion. Br J Neurosurg 1990; 4: 319325 .

26. Porchet, F., Bartanusz, V., Kleinstueck, F.S., Lattig, F., Jeszenszky, D., Grob, D., Mannion, A.F.: Microdiscectomy compared with standard discectomy: an old problem revisited with new outcome measures within the framework of a spine surgical registry. Eur Spine J 2009; Suppl 3: 360366.

27. Riesenburger, R.I., David, C.A.: Lumbar microdiscectomy and microendoscopic discectomy. Minim Invasive Ther Allied Technol 2006; 15: 267-270.

28. Suk, K.S., Lee, H.M., Moon, S.H., Kim, N.H.: Recurrent lumbar disc herniation: results of operative management. Spine 2001; 26: 672-676.

29. Yorimitsu, E., Chiba, K., Toyama, Y., Hirabayashi, K.: Long-term outcomes of standard discectomy for lumbar disc herniation: a follow-up study of more than 10 years. Spine 2001; 26: 652-657.

30. Watters, W.C. $3^{\text {rd, }}$ McGirt, M.J.: An evidence-based review of the literature on the consequences of conservative versus aggressive discectomy for the treatment of primary disc herniation with radiculopathy. Spine J 2009; 9: 240-257.

Martínez Quiñones, J.V.; Aso, J.; Consolini, F.; Arregui, R.: Resultados a largo plazo de la microdiscectomía lumbar en una población laboralmente activa. Neurocirugía 2011; 22; 235-244.

Correpondencia: José Vicente Martínez Quiñones. Servicio de Neurocirugía. Hospital MAZ. Avenida de la Academia General Militar, 74. 50015 Zaragoza

E-mail: chevimq@gmail.com 